$17^{\text {th }}$ International Congress of Metrology, 07005 (2015)

DOI: $10.1051 /$ metrology $/ 201507005$

(C) Owned by the authors, published by EDP Sciences, 2015

\title{
Traceable amount of substance fraction measurements in gases through infrared spectroscopy at PTB
}

\author{
Nils Lüttschwager ${ }^{1}$, Andrea Pogány ${ }^{1}$, Javis Nwaboh ${ }^{1}$, Alexander Klein ${ }^{1}$, Bernhard Buchholz ${ }^{1}$, Olav Werhahn ${ }^{1}$, Volker Ebert ${ }^{1, a}$ \\ ${ }^{1}$ Physikalisch-Technische Bundesanstalt, Bundesallee 100, 38116 Braunschweig, Germany
}

\begin{abstract}
Gas analysis has become an important task in various fields of science and industry, especially through techniques based on laser spectroscopy. Common applications are, for example, the monitoring of the atmosphere to assess human impact on ecosystems and the climate, the monitoring of airborne molecular contamination to supervise sensitive high-tech processes like semiconductor manufacturing, or the analysis of combustion processes. High resolution laser spectroscopy lends itself particularly well for gas analysis, as it is fast, selective, and the results can be made traceable to the SI units. Traceability is of particular importance to maximize the reliability of measurement results, especially if such measurements are the input for complicated models like those used in climate research or if impacting political decisions. In this contribution we give an overview on our approach to achieve traceability of results from spectroscopic amount fraction measurements of $\mathrm{H}_{2} \mathrm{O}, \mathrm{CO}_{2}$, and $\mathrm{NH}_{3}$ including the TILSAM method (traceable infrared laser-spectrometric amount fraction measurement). We discuss how this method applies to tunable diode-laser absorption spectroscopy (TDLAS), cavity ring-down spectroscopy (CRDS), and photoacoustic spectroscopy (PAS). This research is partly embedded in projects within the European Metrology Research Programme (EMRP).
\end{abstract}

\section{Introduction}

The measurement of gas concentrations in terms of the amount fraction is a wide field of gas metrology and can be necessary or beneficial in many fields of science and technology [1]. Several detection schemes exist and depending on the application at hand, there might be clear preferences on which one to choose. Optical detection based on spectroscopy-and in particular laser-based detection techniques-possess several favorable properties which make them the method of choice in many situations like real-time process control [2] or atmospheric monitoring [3]. Some of the advantages are instrument mobility and rapid online detection with minimal disturbance of the investigated gas sample, as well as excellent stability and selectivity. The latter is owned to the fact that each molecular analyte exhibits a unique absorption spectrum which is often and suitably termed its spectroscopic "fingerprint". The absorption signature of an analyte can be quantitatively related to its amount fraction in a gas mixture through fundamental molecular properties, the absorption line parameters. This can enable absolute measurements and lessen tight restrictions to certain matrix gas compositions and physical conditions which may apply to measurement results relying on a calibration with reference gases. Using traceable line parameters, such absolute or "calibration-free" laser spectroscopic measurements can be made traceable to the SI and are the basis for a measurement procedure which we call TILSAM (for traceable infrared laserspectrometric amount fraction measurements, an outcome of the EURAMET project 934 [4]).

Gas spectroscopy has grown to technical maturity and numerous spectrometers based on various measurement principles [1] are now commercially available. However, traceability of the measurement results is often not addressed directly, leading to a lack of comparability. Clearly, there is a need to promote traceability of spectroscopic amount fraction measurements, e.g. by developing optical transfer standards for instrument calibration or by providing traceable absorption line parameters which are needed for instruments which perform absolute measurements. Thus, the development of transfer standards, measurements of traceable line data, and the metrological characterization of commercial spectrometers are key goals of our research.

Well separated absorption lines which are suitable to be employed in TILSAM are easily found for small molecules such as $\mathrm{H}_{2} \mathrm{O}, \mathrm{CO}_{2}$ and $\mathrm{NH}_{3}$, on which we currently concentrate our research. We developed absolute laser hygrometers which are used in atmospheric research [5], combustion research [6,7], or to measure humidity in natural gas or biogas for quality assurance purposes [8]. In the framework of the completed EMRP project MacPoll [9] we developed an absolute spectrometer targeting $\mathrm{CO}_{2}$ and $\mathrm{H}_{2} \mathrm{O}$ to 
validate the $\mathrm{CO}_{2}$ concentration and humidity in zero gas which is used to check the zero-reading of calibrated sensors and as dilution gas for reference gas mixtures [10]. In the currently running EMRP projects MetAMC [11] and MetNH3 [12] we work on reliable online detection of $\mathrm{NH}_{3}$ in clean rooms based on cavity ring-down spectroscopy (CRDS) and photoacoustic spectroscopy (PAS), and on a traceable optical transfer standard for $\mathrm{NH}_{3}$ detection based on a commercial CRDS spectrometer, respectively.

Traceability is a key issue for the above mentioned applications. This is easily understood by the potential harm which could follow from erroneous measurements when political decisions (e.g. concerning $\mathrm{CO}_{2}$ or $\mathrm{NH}_{3}$ emissions), terms of trade (e.g. based on natural gas quality) or yields in semiconductor fabrication (clean room air quality) depend on such measurements. In this contribution we discuss our approach to achieve traceability in laser-based amount of substance fraction measurements and describe the basic measurement principles and how uncertainty and traceability are addressed.

\section{Background}

We measure gas concentrations in terms of the amount of substance fraction, which is defined as the amount of the analyte divided by the total amount of molecules in the gas sample. The amount fraction is a dimensionless quantity and often given in pseudo units like ppm or $\mathrm{ppb}$ which represent powers of ten. We prefer the SI unit "mol/mol" which avoids ambiguities and allows the usage of SI-prefixes [13,14].

The amount fraction can be related to light absorption via the Beer-Lambert law:

$$
\Phi(\widetilde{v})=\Phi_{0}(\widetilde{v}) \cdot \mathrm{e} \quad[\mathrm{x}(\widetilde{\varphi}) \cdot L],
$$

where $\Phi_{0}(\tilde{v})$ is the incident radiant power at wavenumber $\tilde{v}, \Phi(\tilde{v})$ the radiant power transmitted through a sample cell of length $L$, and $k(\tilde{v})$ the absorption coefficient. In the ideal gas approximation, the absorption coefficient is directly proportional to the amount of substance fraction $(x)$ of the associated gas:

$$
\begin{aligned}
k(\tilde{v}) & =S_{T} \cdot r_{\text {iso }} \cdot g\left(\tilde{v}-\widetilde{v}_{0}\right) \cdot n_{i} \\
& =S_{T} \cdot r_{\text {iso }} \cdot g\left(\tilde{v}-\widetilde{v}_{0}\right) \cdot x_{i} \cdot \frac{p}{k_{B} \cdot T} .
\end{aligned}
$$

Here, $S_{T}$ is the line strength at temperature $T, r_{\text {iso }}$ a correction factor (see section 3.1), $p$ the total gas pressure and $k_{B}$ the Boltzmann constant. The line shape of the detected absorption line is approximated by the line shape function $g\left(\tilde{v}-\tilde{v}_{0}\right)$. Its area is normalized such that:

$$
\int_{-\infty}^{+\infty} g\left(\widetilde{v}-\widetilde{v}_{0}\right) \mathrm{d} \widetilde{v}=1
$$

The absorption coefficient may be measured by techniques like tunable diode-laser absorption spectroscopy (TDLAS), CRDS, or PAS. In TDLAS, the absorbance $(A)$ is measured and related to the absorption coefficient via:

$$
A(\widetilde{v})=1 \frac{\Phi_{0}(\widetilde{v})}{\Phi(\widetilde{v})}=k(\widetilde{v}) L .
$$

In CRDS, the ring down-time $\tau(\tilde{v})$ of light oscillating in an optical cavity is related to the absorption coefficient via [15]:

$$
\frac{1}{c}\left(\frac{1}{\tau(\widetilde{v})}-\frac{1}{\tau_{0}(\widetilde{v})}\right)=k(\widetilde{v})
$$

(the index 0 indicates an empty cavity). Finally, the photoacoustic signal $S_{\mathrm{PA}}$ in PAS is proportional to the absorption coefficient and the power of the irradiating laser [16]:

$$
S_{\mathrm{P} A} \propto P \cdot k(\widetilde{v})
$$

\section{Traceability in laser-based measure- ments}

\subsection{TDLAS}

In TDLAS, the model equation which relates the amount fraction $x_{i}$ to the line area $A_{\text {line,TDLAS }}$ of the investigated absorption line is derived by integration of the absorbance spectrum (eq. 2), leading to [4]:

$$
x_{i}=\frac{k_{B} \cdot T}{S_{T} \cdot r_{\text {iso }} \cdot p \cdot L} \cdot A_{\text {line, TDLAS }}
$$

A TDLAS spectrum is recorded by repeatedly scanning the wavelength of a narrow linewidth diode laser over the probed absorption line(s) using a triangular current ramp and measuring the radiant power of light transmitted through an absorption cell which holds the gas sample under study. Temperature and pressure are recorded simultaneously to the TDLAS signals of the gas sample, while the absorption path length is determined prior to TDLAS measurements. Figure 1 shows a raw spectrum of $\mathrm{CO}_{2}$ (diluted in $\mathrm{N}_{2}$ ) and the resulting absorbance spectrum after conversion of the $\mathrm{x}$-axis from sample points (i.e. time) to wavenumbers. The latter is derived from the transmission curve of laser light passing through a Fabry-Pérot etalon [17]. The absorption line area is retrieved via a non-linear least squares fit of the absorbance spectrum using an appropriate line profile (e.g. Voigt) to approximate the measured line shapes. The fit can be restricted by fixing the line width according to available broadening coefficients and the measured pressure and temperature making it more robust. 


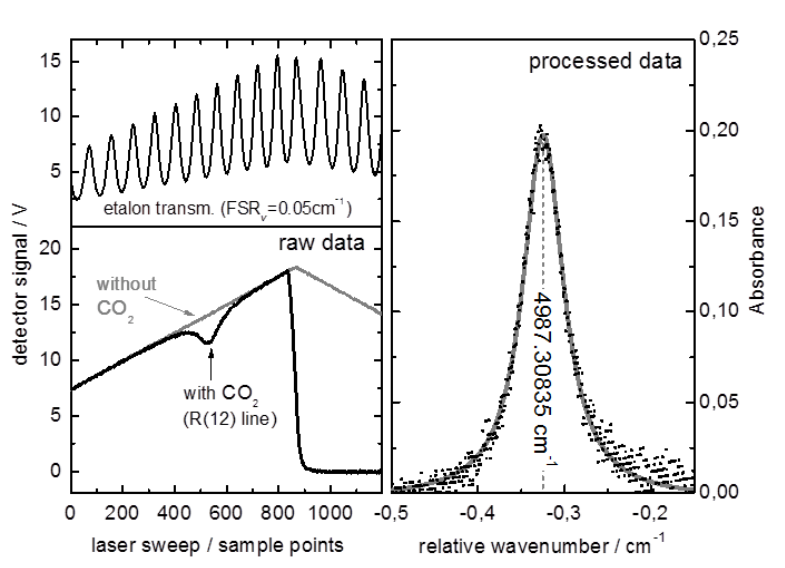

Figure 1: Example of TDLAS raw and processed data (absorbance spectrum of a $\mathrm{CO}_{2}$ line [17])

Traceability of pressure and temperature measurements is accomplished by usage of sensors which are traceable to PTB pressure and temperature standards. A traceable optical path length can be derived by different methods, depending on the type of absorption cell which is used. The length of a single pass absorption cell can be measured by mechanical means, e.g. using a traceable caliper, while for multipass cells we use a traceable laser distance meter. A complementary method which is often used in addition is based on comparative measurements with the same gas mixture and a traceable reference cell [18]. Pressure, temperature and optical path length relative $k=2$ uncertainties in the sub-percent range are routinely achieved and thus are typically not limiting the overall amount fraction measurement uncertainty. Note that we calculate all uncertainties following the GUM [19] and that the coverage factor $k=2$ corresponds to a confidence interval of $95 \%$.

The isotopic correction factor $r_{\text {iso }}$ is used to match the line strength, given for a reference abundance value, to the actual isotopic composition of the sample gas. Usually a natural abundance of the different isotopologues is assumed $\left(r_{\text {iso }}=1\right)$ and the uncertainty is calculated from the uncertainty of the natural abundance of the elements $[20,21]$. In this case, for gases like $\mathrm{CO}_{2}, \mathrm{NH}_{3}$, and $\mathrm{H}_{2} \mathrm{O}$ the relative $k=2$ uncertainty of $r_{\text {iso }}$ is $<0.1 \%$ [10] and does not contribute significantly to the overall uncertainty budget of the amount fraction. However, a parallel or even independent evaluation of the actual isotopic sample composition is desirable.

The uncertainties of the line strength $S_{T}$ and the line area $A_{\text {line,TDLAS }}$ often dominate a TILSAM uncertainty budget. If we measure line strengths by means of TDLAS [22-25] or FTIR spectroscopy [26], relative expanded uncertainties in the $1 \%$ range can be achieved. If line data from spectral databases like HITRAN [27] or GEISA [28] are employed, uncertainties can be considerably higher (typically $5 \%$ to $10 \%$ ) and traceability is missing for the vast majority of lines. Our uncertainty assessment [10] for the line area includes laser tuning (i.e. the wavenumber axis), parasitic absorption [29], laser side mode emission, detector non-linearity and the uncertainty in line fitting. The latter depends on the achieved signal-to-noise level and how well the probed line shape is represented by a Voigt profile, while the other uncertainties can be evaluated empirically in separate measurements [10]. For example, the uncertainty in the wavenumber axis may additionally be estimated by comparing line positions to reference values or experimentally determined Doppler widths of low pressure signals to calculated values based on measured gas temperatures $[22,23]$.

The combined uncertainty achieved in TDLAS measurements referring to the TILSAM protocol is demonstrated in several of our earlier publications. For example, ref. [17] reported measurements from $60 \mathrm{mmol} / \mathrm{mol}$ to $360 \mu \mathrm{m} / \mathrm{mol} \mathrm{CO}_{2}$ in $\mathrm{N}_{2}$ gas mixtures and yielded relative expanded uncertainties in the range $1.4 \%$ to $2.8 \%$, respectively. A study presented in ref. [10] showed that uncertainty figures do not suffer when $\mathrm{CO}_{2}$ and $\mathrm{H}_{2} \mathrm{O}$ amount fraction measurements are combined by scanning the laser wavelength over two neighboring $\mathrm{CO}_{2}$ and $\mathrm{H}_{2} \mathrm{O}$ absorption lines. Here, the relative expanded uncertainties were estimated as $2 \%$ to $3 \%$ for nominal amount fractions of $100 \mu \mathrm{mol} / \mathrm{mol}$, documenting a slight improvement for $\mathrm{CO}_{2}$ upon the earlier work [17]. Finally, ref. [5] documents a relative expanded uncertainty of $4.3 \%$ for an airborne laser hygrometer measuring water concentrations under field situations in the $20 \mathrm{mmol} / \mathrm{mol}$ to $600 \mu \mathrm{m} / \mathrm{mol}$ range. The laser hygrometer was validated against the German primary reference standard for humidity yielding relative deviations $\leq 2.5 \%$ (Figure 2). This shows that TILSAM can provide reliable results with uncertainties in the low percent region while leveraging the benefits of laser spectroscopic measurements.

\subsection{CRDS and PAS}

Our work on traceability in CRDS and PAS amount fraction measurements is still ongoing and we discuss these techniques here just briefly. In principle, the TILSAM method is also applicable to CRDS [30] with the model equation being very similar to the corresponding TDLAS variant (eq. 5):

$$
x_{i}=\frac{k_{B} \cdot T}{S_{T} \cdot r_{\text {iso }} \cdot p} \cdot A_{\text {line, } \mathrm{CRDS}}
$$

The absorption path length $L$ is now contained in the absorption coefficient and does not appear in the model equation. The line area $A_{\text {line, CRDS }}$ is derived from a fit of the CRDS spectrum ${ }^{1}$ in the same way as described above for TDLAS. Traceability of the ring-down time axis must be addressed by using a traceable oscilloscope or data acquisition card and the uncertainty in fitting the ring-down curves must be accounted for. A prerequisite for traceable measurements remains the availability of traceable line data (at least line strength $S_{T}$ ). Measurement of line data is our first step to achieve traceability while working with commercial instruments

\footnotetext{
${ }^{1}$ absorption coefficient vs. wavenumber, see eq. 3
} 


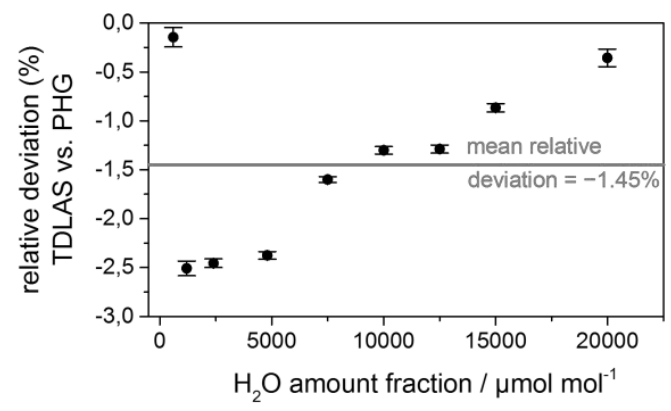

Figure 2: Relative deviations between an absolute TDLAS hygrometer and PTB's primary reference humidity generator $(\mathrm{PHG})$ [5].

intended to be used as optical transfer standard in the MetNH3 project [12].

The signal in PAS is related to the absorption coefficient (and thus the amount fraction) in a more complicated way. The relation of the PA signal $S_{\mathrm{PA}}$ to the absorption coefficient may be expressed as [16]:

$$
S_{\mathrm{PA}}(\widetilde{v})=P \cdot R \cdot[C \cdot \eta \cdot k(\widetilde{v})+B(\widetilde{v})] .
$$

Accordingly, the PA signal depends on numerous influence quantities: the power of the laser radiation $P$, the microphone responsivity $R$, a constant $C$ which is specific to the photoacoustic cell, the efficiency of radiation to heat conversion $\eta$, and the function $B(\tilde{v})$ which describes the wavenumber dependence of the background signal caused by light absorption at cell windows or walls. These influence quantities in turn depend on environmental conditions, gas matrix composition or the modulations scheme (amplitude or wavelength modulation, modulation frequency), such that a quantification and subsequent absolute measurements present significant difficulties. A calibration with traceable reference gases is necessary where the amount fraction can be related to the integral of absorption lines retrieved from a fit. The dependence of the photoacoustic signal on environmental conditions like pressure, temperature and matrix composition of the gas sample should be met by tightly controlling and monitoring these parameters with traceable equipment. This is a satisfactory solution for applications like clean room monitoring [11], where the environmental conditions are rather stable.

\section{Conclusions}

Amount fraction measurements based on laser spectroscopy are versatile and advantageous in a wide field of applications. Emerging commercial instruments are easy to use and do not require specialized knowledge of spectroscopy which makes them attractive tools in gas analysis, but with missing traceability when operated as absolute instruments. Traceability in absolute, laser-based amount fraction measurements is feasible and described by the TILSAM protocol [4]. Absolute TDLAS measurements based on TILSAM have been shown to yield relative expanded uncertainties in the low percent range $[5,17]$ and TILSAM instrumentation may be used to certify gas mixtures or as optical transfer standard [10]. TILSAM can also be applied for absolute measurements based on CRDS [30,31], extending its scope to the measurement of trace concentrations. PAS is another technique well suited for trace gas detection but needs calibration with reference gases and is thus not yet addressed by the TILSAM method.

The TILSAM procedure is currently compared against established methods referring to gaseous standards in the EURAMET project 1280 [32] to test its competitiveness. Discussions on the feasibility of TILSAM-based primary methods in gas metrology are ongoing [33].

\section{Acknowledgement}

Parts of this work were done in the framework of the European Metrology Research Programme (EMRP). The EMRP is jointly funded by the EMRP participating countries within EURAMET and the European Union.

Parts of this work have been funded by the HAI Project (Deutsche Forschungsgemeinschaft, FKZ EB 235/3-1 and EB 235/3-2) within the HALO-SPP 1294.

\section{References}

1. J. Hodgkinson and R. P. Tatam, Meas. Sci. Technol. 24, 012004 (2013).

2. M. Lackner, Rev. Chem. Eng. 23, (2007).

3. T. Gardiner, M. I. Mead, S. Garcelon, R. Robinson, N. Swann, G. M. Hansford, P. T. Woods, and R. L. Jones, Rev. Sci. Instrum. 81, 083102 (2010). 4. O. Werhahn and J. C. Petersen, TILSAM-MethodDraft A of a Technical Protocol-, http://www.euramet.org/fileadmin/docs/projects/934_M ETCHEM_Interim_Report.pdf, (2010).

5. B. Buchholz, N. Böse, and V. Ebert, Appl. Phys. B 116, 883 (2014).

6. O. Witzel, A. Klein, C. Meffert, S. Wagner, S. Kaiser, C. Schulz, and V. Ebert, Opt. Express 21, 19951 (2013).

7. O. Witzel, A. Klein, C. Meffert, C. Schulz, S. A. Kaiser, and V. Ebert, Proc. Combust. Inst. 35, 3653 (2015).

8. J. G. Gallegos, R. Benyon, S. Avila, A. Benito, R. M. Gavioso, H. Mitter, S. Bell, M. Stevens, N. Böse, V. Ebert, M. Heinonen, H. Sairanen, A. Peruzzi, R.

Bosma, and M. Val'ková, J. Nat. Gas Sci. Eng. 23, 407 (2015)

9. A. Baldan, 16th Int. Congr. Metrol. 10002 (2013).

10. A. Pogány, S. Wagner, O. Werhahn, and V. Ebert, Appl. Spectrosc. 69, 257 (2015).

11. http://www.ptb.de/emrp/metamc.html (accessed June 2015).

12. http://www.metnh3.eu/ (accessed June 2015).

13. T. J. Quinn and I. M. Mills, Metrologia 35, 807 (1998).

14. R. J. C. Brown and P. J. Brewer, Metrologia 52, L1 (2015). 
15. G. Berden, R. Peeters, and G. Meijer, Int. Rev. Phys. Chem. 19, 565 (2000).

16. Z. Bozóki, A. Pogány, and G. Szabó, Appl. Spectrosc. Rev. 46, 1 (2011).

17. J. A. Nwaboh, O. Werhahn, P. Ortwein, D. Schiel, and V. Ebert, Meas. Sci. Technol. 24, 015202 (2013).

18. J. A. Nwaboh, O. Witzel, A. Pogány, O. Werhahn, and V. Ebert, Int. J. Spectrosc. 2014, 132607 (2014).

19. JCGM 100:2008, Evaluation of Measurement Data

- Guide to the Expression of Uncertainty in

Measurement (GUM),

http://www.bipm.org/en/publications/guides/gum.html, (2008).

20. M. Berglund and M. E. Wieser, Pure Appl. Chem. 83, 397 (2011).

21. M. E. Wieser and T. B. Coplen, Pure Appl. Chem. 83, 359 (2010).

22. A. Pogány, O. Ott, O. Werhahn, and V. Ebert, J. Quant. Spectrosc. Radiat. Transf. 130, 147 (2013).

23. J. A. Nwaboh, O. Werhahn, and V. Ebert, Mol. Phys. 112, 2451 (2014).

24. G. J. Padilla-Viquez, J. Koelliker-Delgado, O. Werhahn, K. Jousten, and D. Schiel, IEEE Trans. Instrum. Meas. 56, 529 (2007).

25. A. Pogány, A. Klein, and V. Ebert, J. Quant. Spectrosc. Radiat. Transf. (in press).

26. G. Li, A. Serdyukov, M. Gisi, O. Werhahn, and V. Ebert, J. Quant. Spectrosc. Radiat. Transf. (in press).

27. L. S. Rothman, I. E. Gordon, Y. Babikov, A. Barbe, D. Chris Benner, P. F. Bernath, M. Birk, L. Bizzocchi,

V. Boudon, L. R. Brown, A. Campargue, K. Chance, E. A. Cohen, L. H. Coudert, V. M. Devi, B. J. Drouin, A. Fayt, J.-M. Flaud, R. R. Gamache, J. J. Harrison, J.-M. Hartmann, C. Hill, J. T. Hodges, D. Jacquemart, A. Jolly, J. Lamouroux, R. J. Le Roy, G. Li, D. A. Long, O. M. Lyulin, C. J. Mackie, S. T. Massie, S.

Mikhailenko, H. S. P. Müller, O. V. Naumenko, A. V. Nikitin, J. Orphal, V. Perevalov, A. Perrin, E. R. Polovtseva, C. Richard, M. A. H. Smith, E. Starikova, K. Sung, S. Tashkun, J. Tennyson, G. C. Toon, V. G. Tyuterev, and G. Wagner, J. Quant. Spectrosc. Radiat. Transf. 130, 4 (2013).

28. N. Jacquinet-Husson, L. Crepeau, R. Armante, C. Boutammine, A. Chédin, N. A. Scott, C. Crevoisier, V. Capelle, C. Boone, N. Poulet-Crovisier, A. Barbe, A. Campargue, D. Chris Benner, Y. Benilan, B. Bézard, V. Boudon, L. R. Brown, L. H. Coudert, A. Coustenis, V. Dana, V. M. Devi, S. Fally, A. Fayt, J.-M. Flaud, A. Goldman, M. Herman, G. J. Harris, D. Jacquemart, A. Jolly, I. Kleiner, A. Kleinböhl, F. Kwabia-Tchana, N. Lavrentieva, N. Lacome, L.-H. Xu, O. M. Lyulin, J.-Y. Mandin, A. Maki, S. Mikhailenko, C. E. Miller, T. Mishina, N. Moazzen-Ahmadi, H. S. P. Müller, A. Nikitin, J. Orphal, V. Perevalov, A. Perrin, D. T. Petkie, A. Predoi-Cross, C. P. Rinsland, J. J. Remedios, M. Rotger, M. A. H. Smith, K. Sung, S. Tashkun, J. Tennyson, R. A. Toth, A.-C. Vandaele, and J. Vander Auwera, J. Quant. Spectrosc. Radiat. Transf. 112, 2395 (2011).

29. B. Buchholz and V. Ebert, Meas. Sci. Technol. 25, 075501 (2014).
30. O. Werhahn, BA-TILSAM Method - a Technical Description -,

https://www.ptb.de/cms/fileadmin/internet/fachabteilun gen/abteilung_3/3.2_analytische_messtechnik_und_dru ck/3.22/IMERA_Plus.pdf, (2011).

31. J. A. Nwaboh, S. Persijn, K. Heinrich, M. Sowa, P. Hering, and O. Werhahn, Int. J. Spectrosc. 2012, 894841 (2012).

32. http://www.euramet.org/technicalcommittees/search-tc-projects/ (accessed June 2015). 33. M. J. T. Milton and T. J. Quinn, Metrologia 38, 289 (2001). 